treatment of 0 days. In the standard-care period, $40 / 41(97.6 \%$, 95\% CI: 87.1-99.9) received treatment; 15 (37.5\%) were treated presumptively on the same day (due to symptoms/risk) and $25(62.5 \%)$ were treated on the basis of the laboratory test result; and of these $44 \%$ were treated in 1-7 days, $44 \%$ in $8-14$ days and $12 \%$ in $15+$ days, with a median time-to-treatment of 8 days.

Conclusion This site is already part of a strong and comprehensive STI control program run by NHC over 20 years. Early findings from TTANGO show further improvement in STI management were achieved with point-of-care tests, with treatment occurring on average 8 days sooner for those treated on the basis of a test result. Future analyses will include all 12 clinics and also assess if re-infections have reduced.

Disclosure of interest statement No pharmaceutical grants were received in the development of this study. TTANGO is funded by a NHMRC project grant. The GeneXpert cartridges were purchased from Cepheid and Cepheid provided machines on loan for the duration of the study.

\section{P07.05 UTILITY OF POOLED URINE SAMPLES FOR DETECTION OF CHLAMYDIA TRACHOMATIS INFECTION IN ASYMPTOMATIC PREGNANT WOMEN IN NORTHERN INDIA}

${ }^{1} \mathrm{~S}$ Sethi*, ${ }^{1}$ A Roy, ${ }^{2}$ R Bagga, ${ }^{3}$ SD Mehta. ${ }^{1}$ Department of Medical Microbiology, General Hospital, Chandigarh; 'Department of Obstertics and Gynaecology, General Hospital, Chandigarh; ${ }^{3}$ Postgraduate Institute of Medical Education and Research Chandigarh, Department of Dermatology, General Hospital, Chandigarh

\subsection{6/sextrans-2015-052270.321}

Background Urogenital infections by Chlamydia trachomatis (CT) are the most prevalent sexually-transmitted bacterial diseases in women. Cost effective screening of women for C. trachomatis infection in developing countries is highly desirable for reducing morbidity and complications like pelvic inflammatory diseases, ectopic pregnancy and infertility. Results of noninvasive urine samples tests for C. trachomatis are nearly identical to samples collected directly from the cervix or urethra. The present study used the pooled urine samples to decrease the cost of screening for C. trachomatis.

Methods First void urine samples were collected from 1000 asymptomatic pregnant women having gestational age less than 24 weeks attending the Antenatal Clinics at PGIMER, Chandigarh during July 2009 to June 2012. The pooled urine samples (5x pooled processed specimen) were tested for presence of $C$. trachomatis by Amplicor CT PCR kit (Roche Diagnostic) and positive results were further tested separately on each urine sample. Direct fluroscent antibody test (DFA) assay was used on urine specimen which were positive by PCR to confirm the positive results.

Results Overall C. trachomatis infection tested by both PCR and DFA was present in $1.6 \%(16 / 1000)$ of asymptomatic pregnant women. A total of 200 pools of urine samples were tested and 20 pools were positive for C. trachomatis. When these pools were tested individually, 20 (10\%) samples were positive for CT (In one pool, 2 samples were positive and one pool was false positive). Four samples were negative by DFA. Pooling of urine samples saved $70 \%$ of reagent costs in our study.

Conclusions The study shows C. trachomatis infection was present in $1.6 \%$ of pregnant women which indicates low prevalence of infection in northern India. Pooling of urine samples saved labour, cost (70\% reduction) and time in screening large number of samples in resource-limited settings.

Disclosure of interest statement None (No conflict of interest). The study was funded by Indian Council of Medical Research, New Delhi. No pharmaceutical grants were received in the development of this study.

\section{P07.06 A LOW COST, HAND-HELD POINT OF CARE MOLECULAR DIAGNOSTIC DEVICE FOR SEXUALLY TRANSMITTED INFECTIONS}

${ }^{1}$ RE Mackay ${ }^{*},{ }^{1} M$ Branavan, ${ }^{1,2} \mathrm{P}$ Craw, ${ }^{1}$ A Naveenathayalan, ${ }^{3}$ ST Sadiq, ${ }^{1}$ W Balachandran. ${ }^{1}$ Brunel University London; ${ }^{2}$ Commonwealth Scientific and Industrial Research Organisation, Hobart; ${ }^{3}$ St. George's University of London

\subsection{6/sextrans-2015-052270.322}

Introduction Rapid and accurate field diagnostics have potential to impact on the burden of STIs in resource poor settings. Microfluidic and nano technologies offer opportunities to create molecular detection platforms but costs may be prohibitive. We present a low cost isothermal amplification, point of care test for rapid identification of sexually transmitted infections. Sample collection integrates directly with a microfluidic device for automated sample preparation, isothermal amplification and optical detection.

Methods Cell lysis, within the microfluidic cartridge, is conducted using a chemical method and nucleic acid purification is achieved on activated cellulose membrane. The microfluidic device incorporates passive mixing of lysis-binding buffers and sample using a serpentine channel. Isothermal amplification is conducted using thermophillic helicase dependent amplification (tHDA) and recombinase polymerase amplification (RPA). A low cost real-time isothermal amplification platform has been developed capable of running six amplifications simultaneously.

Results Results have shown extraction efficiencies for the new membrane of $69 \%$ and $57 \%$ compared to commercial Qiagen extraction of $85 \%$ and $59.4 \%$ for $0.1 \mathrm{ng} / \mu \mathrm{L}$ and $100 \mathrm{ng} / \mu \mathrm{L}$ salmon sperm DNA respectively spiked in phosphate buffered solution. Extraction experiments using the serpentine passive mixer cartridges incorporating lysis and nucleic acid purification showed extraction efficiency around $80 \%$ of the commercial Qiagen kit. The platform is capable of detecting $1.32 \times 10^{6}$ copies of target DNA through thermophillic helicase dependent amplification and $1 \times 10^{5}$ copies of Chlamydia trachomatis genomic DNA within 10 min through RPA.

Conclusion We have produced a low cost, rapid nucleic acid extraction, isothermal amplification and detection platform consistent with use remote resource poor settings. The simple optics setup demonstrated high sensitivity and rapid detection of the tHDA and RPA reactions removing the requirement for expensive dichroic filters and lenses. Diagnostic performance of the device is currently being undertaken.

Disclosure of interest statement No Disclosure of interest.

\section{P07.07 MULTIPLEXING STI CAUSING PATHOGENS USING MNAZYME QPCR: A NOVEL REAL-TIME TECHNOLOGY WITH A SUPERIOR CAPACITY FOR MULTIPLEXING}

SM Erskine, L Tan, S Walker, C Denver, E Mokany*. SpeeDx Pty Ltd, National Innovation Centre, Eveleigh, Australia

10.1136/sextrans-2015-052270.323 
Introduction The pressure for faster, cheaper, better sample testing has resulted in an increasing need for multiplexed assays. Current real-time chemistries are expensive and/or difficult to multiplex. MNAzyme qPCR is a novel real-time technology that provides a powerful tool for molecular diagnostics with multiple advantages over other real-time chemistries. A multiplex MNAzyme qPCR panel was used for the identification of causative bacterial pathogens important for prompt and proper treatment of STIs.

Methods An MNAzyme qPCR panel was developed for the multiplexed detection of Mycoplasma genitalium, Mycoplasma hominis, Ureaplasma parvum and Ureaplasma urealyticum together with a DNA extraction control. MNAzymes are comprised of two DNA partzymes, which come together only in the presence of PCR amplicons, to form active enzymes that modify universal reporter probes and produce signals that are monitored in realtime. Since signal production requires the binding of two partzymes and two PCR primers to a target, this approach has higher specificity than other chemistries, making it ideal for molecular diagnostics. The use of a series of well-characterised, universal probes provides many advantages over target specific probes. They result in reliable, consistent performance when coupled to any target and facilitate rapid and simple development of multiplex tests.

Results The STI panel showed robust performance in multiplex with high analytical specificity and sensitivity. The $\mathrm{R}^{2}$ for all targets was above 0.99 and PCR efficiencies ranged from $90 \%$ to $110 \%$. No inter-panel cross-reactivity was observed and no cross-reactivity was detected using a wide range of non-target organisms.

Conclusion MNAzyme qPCR provides a superior approach to real-time PCR with the advantages of greater specificity, robust multiplex performance, reduced cost, and it is more amenable than other technologies for the rapid development of multiplex assays. These features have been demonstrated through the STI MNAzyme qPCR panel.

Disclosure of interest statement SpeeDx is the developer and manufacturer of the assay evaluated in this study.

\section{P07.08 DESIGNING OF MOLECULAR BEACON BASED POLYMERASE CHAIN REACTION METHOD AS AN UNCONVENTIONAL LOW COST DIAGNOSTIC ASSAY FOR SEXUALLY TRANSMITTED DISEASES}

D Sachdev, SC Sonkar, AL Patel, D Saluja*. Dr B R Ambedkar Center for Biomedical Research, University of Delhi, Delhi-110007, India

\subsection{6/sextrans-2015-052270.324}

Introduction About 300 million new infections of gonorrhoea, Chlamydia, or trichomoniasis occur each year. Young adults, population with health inequities and people in resource poor settings bear the significant proportion of sexually transmitted infections (STI). Therefore, rapid, inexpensive and acceptable tests are needed to address STIs in developing countries. PCR based tests are recommended for diagnosis of several STIs, but such tests require sophisticated infrastructure, need to be imported and hence cannot be used in resource limited settings. Affordability can be countered by the development of in house diagnostic assays which are practical and easy to use, and produced within country to further lower the cost.
Methods Endocervical swabs were collected from patients visiting gynaecology department of hospitals in Delhi. In-house PCR based assay was developed for C. trachomatis, N. gonorrhoeae and $T$. vaginalis and modified to visual assay using molecular beacon for end-point detection.

Results The molecular beacon based PCR assay was developed for C. trachomatis, N. gonorrhoeae and T. vaginalis and evaluated against different commercial kits viz; Roche AMPLICOR NG/CT kit and qPCR kit of Abbott and fast-track diagnostics. Specificity of molecular beacon was confirmed by competition experiments. Diagnostic tests were more than $95 \%$ specific and 99.0\% sensitive for all the three pathogens and negative and positive predicted values were around $98.5 \%$ and $97.5 \%$, respectively at $95 \%$ CI for these three pathogens. We also observed that dry swab samples gave concordant results with that of wet swabs. Assay reagents were stable for more than 6 months at room temperature. We also report, high rates of co-infection for C. trachomatis + N. gonorrhoeae, C. trachomatis + T. vaginalis, N. gonorrhoeae + T. vaginalis amongst women patients in India. Conclusions Development of a rapid, sensitive, specific and PCR based visual diagnostic assay, suitable for developing countries will provide a better disease management.

\section{P07.09 A VALIDATION STUDY OF THE CEPHEID XPERTÂ CT/NG FOR DETECTING CHLAMYDIA TRACHOMATIS AND NEISSERIA GONORRHOEAE IN RECTAL SAMPLES}

${ }^{1}$ LA Cosentino*, ${ }^{2} \mathrm{CS}$ Danby, 'LK Rabe, ${ }^{1} \mathrm{CL}$ Priest, ${ }^{1} \mathrm{KC}$ Damare, 'IS Macio, 'LA Meyn, ${ }^{1,2} \mathrm{HC}$ Wiesenfeld, 1,2 $\mathrm{SL}$ Hillier. 'Magee-Womens Research Institute; ${ }^{2}$ The University of Pittsburgh, Department of Obstetrics, Gynecology and Reproductive Sciences, Pittsburgh, $P A, U S A$

\subsection{6/sextrans-2015-052270.325}

Introduction The objective of this study was to compare the Cepheid Xpert CT/NG (Xpert) and Gen-Probe Aptima (AC2) systems for the identification of Chlamydia trachomatis (CT) and Neisseria gonorrhoeae (GC) from rectal swabs.

Methods Anorectal swabs were collected from 399 participants (224 men and 175 women) who reported a lifetime history of anal receptive intercourse. True positives for this study were defined if both AC2 and Xpert were positive, or if either AC2 or Xpert were positive and the APTIMA CT or APTIMA GC, which target alternate primers, were positive.

Results There was a high level of agreement between Xpert and AC2 for detection of CT and GC from rectal swabs, with only 2/30 discrepant results for GC and 9/59 discrepant results for CT. Following resolution of discrepants, rectal swabs were positive for CT in $58(14.5 \%)$ and for GC in $30(7.5 \%)$ of the 399 participants. For CT, the sensitivity and specificity of Xpert (96.6\%, 99.7\%) and AC2 (91.4\%, 100\%) were comparable, with men and women both having high frequencies of rectal CT $(17.4 \%$ vs $10.9 \%, \mathrm{P}=0.08)$. For $\mathrm{GC}$, the sensitivity and specificity of Xpert $(100 \%, 100 \%)$ and AC2 $(93.3 \%, 100 \%)$ were similar. However, rectal GC was detected much more frequently in men than in women $(11.6 \%$ vs $2.3 \%, \mathrm{P}<0.001)$.

Conclusion Rectal GC and CT were common among people reporting a history of receptive anal sex. Xpert and AC2 had high levels of agreement for rectal swab samples.

Disclosure of interest statement Reagents for CT/GC testing were provided for by Cepheid and Hologic. 